\title{
7. Environmental conditions of the study area
}

\section{$\operatorname{AUTHOR}(S):$}

OHGAKI, SHUN-ICHI; KOMEMOTO, KEN-ICHI; FUNAYAMA, NOBUTAKA

\section{CITATION:}

OHGAKI, SHUN-ICHI ...[et al]. 7. Environmental conditions of the study area. Publications of the Seto Marine Biological Laboratory. Special Publication Series 2011, 11: 178-188

\section{ISSUE DATE:}

2011

URL:

http://hdl.handle.net/2433/159493

RIGHT: 
7. Environmental conditions of the study area

\section{7-1. Height}

Table 4.

The height above the chart datum of each quadrat (QD, see Fig. 1d) was measured in April 1986. For each quadrat, the height at the four corners (NE, north-east; SE, south-east; NW, north-west; SW, south-west) is shown. The point of one corner belongs to 1-4 quadrats, and thus the same value often appears in the rows of different quadrats (e.g., SW of A1, NW of A2, SE of B1, and NE of B2). 


\begin{tabular}{|c|c|c|c|c|c|c|c|c|c|c|c|}
\hline \multicolumn{12}{|c|}{ Table 4} \\
\hline $\mathrm{QD}$ & $\mathrm{NE}$ & $\mathrm{SE}$ & NW & SW & Mean & $\mathrm{QD}$ & $\mathrm{NE}$ & $\mathrm{SE}$ & NW & SW & Mean \\
\hline A 1 & 141 & 121 & 166 & 150 & 145 & E 1 & 225 & 147 & & 155 & 176 \\
\hline A 2 & 121 & 129 & 150 & 123 & 131 & E 2 & 147 & 153 & 155 & 150 & 151 \\
\hline A 3 & 129 & 152 & 123 & 140 & 136 & E 3 & 153 & 151 & 150 & 156 & 153 \\
\hline A 4 & 152 & 150 & 140 & & 147 & E 4 & 151 & 139 & 156 & 142 & 147 \\
\hline A 5 & 150 & 155 & & 158 & 154 & E 5 & 139 & 115 & 142 & 83 & 120 \\
\hline A 6 & 155 & 134 & 158 & 157 & 151 & E 6 & 115 & 139 & 83 & 107 & 111 \\
\hline A 7 & 134 & 148 & 157 & 168 & 152 & E 7 & 139 & 138 & 107 & 106 & 123 \\
\hline A 8 & 148 & 29 & 168 & 159 & 126 & E 8 & 138 & 127 & 106 & 102 & 118 \\
\hline B 1 & 166 & 150 & 121 & 104 & 135 & E 9 & 127 & 159 & 102 & 100 & 122 \\
\hline B 2 & 150 & 123 & 104 & 117 & 124 & E 10 & 159 & 102 & 100 & 78 & 110 \\
\hline B 3 & 123 & 140 & 117 & 144 & 131 & E 11 & 102 & 93 & 78 & & 91 \\
\hline B 4 & 140 & & 144 & 152 & 145 & E 12 & 93 & 82 & & 7 & 61 \\
\hline B 5 & & 158 & 152 & 163 & 158 & E 13 & 82 & 64 & 7 & & 51 \\
\hline B 6 & 158 & 157 & 163 & 167 & 161 & F 1 & & 155 & 159 & 135 & 150 \\
\hline B 7 & 157 & 168 & 167 & 157 & 162 & F 2 & 155 & 150 & 135 & 141 & 145 \\
\hline B 8 & 168 & 159 & 157 & 161 & 161 & F 3 & 150 & 156 & 141 & 133 & 145 \\
\hline B 9 & 159 & & 161 & 106 & 142 & F 4 & 156 & 142 & 133 & 68 & 125 \\
\hline B 10 & & & 106 & 87 & 97 & F 5 & 142 & 83 & 68 & 29 & 81 \\
\hline C 1 & 121 & 104 & 168 & 138 & 133 & F 6 & 83 & 107 & 29 & 73 & 73 \\
\hline C 2 & 104 & 117 & 138 & 139 & 125 & F 7 & 107 & 106 & 73 & 64 & 88 \\
\hline C 3 & 117 & 144 & 139 & 157 & 139 & F 8 & 106 & 102 & 64 & 43 & 79 \\
\hline C 4 & 144 & 152 & 157 & 154 & 152 & F 9 & 102 & 100 & 43 & 39 & 71 \\
\hline C 5 & 152 & 163 & 154 & 157 & 157 & F 10 & 100 & 78 & 39 & & 72 \\
\hline C 6 & 163 & 167 & 157 & 152 & 160 & & & & & & \\
\hline C 7 & 167 & 157 & 152 & 132 & 152 & & & & & & \\
\hline C 8 & 157 & 161 & 132 & 152 & 151 & & & & & & \\
\hline C 9 & 161 & 106 & 152 & 130 & 137 & & & & & & \\
\hline C 10 & 106 & 87 & 130 & 122 & 111 & & & & & & \\
\hline C 11 & 87 & 93 & 122 & 106 & 102 & & & & & & \\
\hline C 12 & 93 & 20 & 106 & 113 & 83 & & & & & & \\
\hline C 13 & 20 & 39 & 113 & 97 & 67 & & & & & & \\
\hline C 14 & 39 & & 97 & 40 & 59 & & & & & & \\
\hline D 1 & 168 & 138 & 225 & 147 & 170 & & & & & & \\
\hline D 2 & 138 & 139 & 147 & 153 & 144 & & & & & & \\
\hline D 3 & 139 & 157 & 153 & 151 & 150 & & & & & & \\
\hline D 4 & 157 & 154 & 151 & 139 & 150 & & & & & & \\
\hline D 5 & 154 & 157 & 139 & 115 & 141 & & & & & & \\
\hline D 6 & 157 & 152 & 115 & 139 & 141 & & & & & & \\
\hline D 7 & 152 & 132 & 139 & 138 & 140 & & & & & & \\
\hline D 8 & 132 & 152 & 138 & 127 & 137 & & & & & & \\
\hline D 9 & 152 & 130 & 127 & 159 & 142 & & & & & & \\
\hline D 10 & 130 & 122 & 159 & 102 & 128 & & & & & & \\
\hline D 11 & 122 & 106 & 102 & 93 & 106 & & & & & & \\
\hline D 12 & 106 & 113 & 93 & 82 & 99 & & & & & & \\
\hline D 13 & 113 & 97 & 82 & 69 & 90 & & & & & & \\
\hline D 14 & 97 & 40 & 69 & & 69 & & & & & & \\
\hline
\end{tabular}




\section{7-2. Water flow}

Table 5.

The intensity of water flow in each quadrat (QD, see Fig. 1d). Water flow was measured as the percentage loss of weight of a plaster lump (120-130 g) fixed at each corner of a quadrat (NE, north-east; SE, south-east; NW, north-west; SW, south-west) during $24 \mathrm{~h}$ in a spring tide (April 26-27, 2005). The point of one corner belongs to 1-4 quadrats and thus the same value often appears in the rows of different quadrats. 


\begin{tabular}{|c|c|c|c|c|c|c|c|c|c|c|c|}
\hline \multicolumn{12}{|c|}{ Table 5} \\
\hline $\mathrm{QD}$ & $\mathrm{NE}$ & SE & NW & SW & Mean & $\mathrm{QD}$ & $\mathrm{NE}$ & SE & NW & SW & Mean \\
\hline A 1 & & 12 & 12 & 11 & 12 & E 1 & & 11 & & 15 & 13 \\
\hline A 2 & 12 & 12 & 11 & 10 & 11 & E 2 & 11 & 13 & 15 & 13 & 13 \\
\hline A 3 & 12 & 11 & 10 & 11 & 11 & E 3 & 13 & 13 & 13 & 13 & 13 \\
\hline A 4 & 11 & 10 & 11 & & 11 & E 4 & 13 & 17 & 13 & 20 & 16 \\
\hline A 5 & 10 & 11 & & 9 & 10 & E 5 & 17 & 17 & 20 & 19 & 18 \\
\hline A 6 & 11 & 16 & 9 & 10 & 12 & E 6 & 17 & 19 & 19 & 20 & 19 \\
\hline A 7 & 16 & 26 & 10 & 17 & 17 & E 7 & 19 & 25 & 20 & 26 & 23 \\
\hline A 8 & 26 & & 17 & 20 & 21 & E 8 & 25 & 22 & 26 & 30 & 26 \\
\hline B 1 & 12 & 11 & & & 12 & E 9 & 22 & 33 & 30 & 36 & 30 \\
\hline B 2 & 11 & 10 & & & 11 & E 10 & 33 & 28 & 36 & 37 & 34 \\
\hline B 3 & 10 & 11 & & 11 & 11 & E 11 & 28 & 32 & 37 & & 32 \\
\hline B 4 & 11 & & 11 & 11 & 11 & E 12 & 32 & 50 & & & 41 \\
\hline B 5 & & 9 & 11 & 12 & 11 & E 13 & 50 & 100 & & & 75 \\
\hline B 6 & 9 & 10 & 12 & 14 & 11 & F 1 & & 15 & 15 & 11 & 14 \\
\hline B 7 & 10 & 17 & 14 & 15 & 14 & F 2 & 15 & 13 & 11 & 15 & 14 \\
\hline B 8 & 17 & 20 & 15 & 18 & 18 & F 3 & 13 & 13 & 15 & 16 & 14 \\
\hline B 9 & 20 & & 18 & 33 & 24 & F 4 & 13 & 20 & 16 & 28 & 19 \\
\hline B 10 & & & 33 & 29 & 31 & F 5 & 20 & 19 & 28 & 18 & 21 \\
\hline C 1 & & & 12 & 10 & 11 & F 6 & 19 & 20 & 18 & 20 & 19 \\
\hline C 2 & & & 10 & 11 & 11 & F 7 & 20 & 26 & 20 & 23 & 22 \\
\hline C 3 & & 11 & 11 & 12 & 11 & F 8 & 26 & 30 & 23 & 28 & 27 \\
\hline C 4 & 11 & 11 & 12 & 13 & 12 & F 9 & 30 & 36 & 28 & & 31 \\
\hline C 5 & 11 & 12 & 13 & 16 & 13 & F 10 & 36 & 37 & & & 37 \\
\hline C 6 & 12 & 14 & 16 & 17 & 15 & & & & & & \\
\hline C 7 & 14 & 15 & 17 & 17 & 16 & & & & & & \\
\hline C 8 & 15 & 18 & 17 & 24 & 19 & & & & & & \\
\hline C 9 & 18 & 33 & 24 & 38 & 28 & & & & & & \\
\hline C 10 & 33 & 29 & 38 & 38 & 35 & & & & & & \\
\hline C 11 & 29 & 40 & 38 & 59 & 42 & & & & & & \\
\hline C 12 & 40 & & 59 & 64 & 54 & & & & & & \\
\hline C 13 & & & 64 & 100 & 82 & & & & & & \\
\hline C 14 & & & 100 & & 100 & & & & & & \\
\hline D 1 & 12 & 10 & & 11 & 11 & & & & & & \\
\hline D 2 & 10 & 11 & 11 & 13 & 11 & & & & & & \\
\hline D 3 & 11 & 12 & 13 & 13 & 12 & & & & & & \\
\hline D 4 & 12 & 13 & 13 & 17 & 14 & & & & & & \\
\hline D 5 & 13 & 16 & 17 & 17 & 16 & & & & & & \\
\hline D 6 & 16 & 17 & 17 & 19 & 17 & & & & & & \\
\hline D 7 & 17 & 17 & 19 & 25 & 20 & & & & & & \\
\hline D 8 & 17 & 24 & 25 & 22 & 22 & & & & & & \\
\hline D 9 & 24 & 38 & 22 & 33 & 29 & & & & & & \\
\hline D 10 & 38 & 38 & 33 & 28 & 34 & & & & & & \\
\hline D 11 & 38 & 59 & 28 & 32 & 39 & & & & & & \\
\hline D 12 & 59 & 64 & 32 & 50 & 51 & & & & & & \\
\hline D 13 & 64 & 100 & 50 & 100 & 79 & & & & & & \\
\hline D 14 & 100 & & 100 & 100 & 100 & & & & & & \\
\hline
\end{tabular}


7-3. Pool coverage

Table 6.

The coverage with pools in each quadrat (QD; Fig. 1d) during April or May from 2005 to 2010 . Coverage was estimated visually at low tide and assigned to one of the following four classes: $1(<25 \%), 2(25-50 \%), 3(50-75 \%)$, or $4(>75 \%)$. -, no data. 


\begin{tabular}{|c|c|c|c|c|c|c|c|c|c|c|c|c|c|}
\hline \multicolumn{14}{|c|}{ Table 6} \\
\hline \multicolumn{14}{|c|}{\begin{tabular}{|l|l|l|l|l|l|l|} 
QD & 2005 & 2006 & 2007 & 2008 & 2009 & 2010 \\
\end{tabular}} \\
\hline A 1 & 1 & 1 & 1 & 1 & 1 & 1 & E 1 & 1 & 1 & 1 & 1 & 2 & 2 \\
\hline A 2 & 2 & 2 & 2 & 2 & 2 & 2 & E 2 & 1 & 2 & 1 & 1 & 1 & 1 \\
\hline A 3 & 2 & 2 & 2 & 2 & 2 & 2 & E 3 & 1 & 1 & 2 & 1 & 1 & 2 \\
\hline A 4 & 3 & 2 & 2 & 2 & 2 & 2 & E 4 & 1 & 1 & 1 & 1 & 1 & 1 \\
\hline A 5 & 3 & 3 & 1 & 3 & 1 & 3 & E 5 & 1 & 1 & 1 & 1 & 1 & 1 \\
\hline A 6 & 3 & 3 & 3 & 2 & 3 & 3 & E 6 & 1 & 1 & 1 & 1 & 1 & 1 \\
\hline A 7 & 2 & 2 & 2 & 2 & 2 & 2 & E 7 & 1 & 1 & 1 & 2 & 1 & 1 \\
\hline A 8 & 2 & 1 & 1 & 1 & 1 & 1 & E 8 & 2 & 2 & 2 & 2 & 2 & 1 \\
\hline B 1 & 3 & 2 & 2 & 3 & 3 & 1 & E 9 & 1 & 1 & 2 & 1 & 1 & 1 \\
\hline B 2 & 2 & 3 & 3 & 3 & 3 & 3 & E 10 & 1 & 1 & 2 & 1 & 1 & 1 \\
\hline B 3 & 4 & 4 & 4 & 4 & 4 & 4 & E 11 & 1 & 2 & 1 & 1 & 2 & 1 \\
\hline B 4 & 2 & 3 & 2 & 3 & 2 & 3 & E 12 & 1 & 1 & 1 & 1 & 1 & 1 \\
\hline B 5 & 1 & 2 & 1 & 2 & 1 & 2 & E 13 & 1 & 1 & 1 & 1 & - & 1 \\
\hline B 6 & 2 & 2 & 2 & 2 & 1 & 2 & F 1 & 1 & 1 & 1 & 1 & 1 & 1 \\
\hline B 7 & 2 & 2 & 2 & 1 & 1 & 2 & F 2 & 1 & 1 & 1 & 1 & 1 & 1 \\
\hline B 8 & 1 & 1 & 2 & 2 & 1 & 2 & F 3 & 1 & 1 & 1 & 1 & 1 & 1 \\
\hline B 9 & 1 & 1 & 3 & 1 & 1 & 1 & F 4 & 1 & 1 & 1 & 1 & 1 & 1 \\
\hline B 10 & 1 & 1 & 1 & 1 & 1 & 1 & F 5 & 1 & 1 & 1 & 1 & 1 & 1 \\
\hline C 1 & 3 & 3 & 3 & 3 & 2 & 2 & F 6 & 1 & 1 & 1 & 1 & 1 & 1 \\
\hline C 2 & 4 & 4 & 4 & 3 & 3 & 3 & F 7 & 1 & 1 & 1 & 1 & 1 & 1 \\
\hline C 3 & 3 & 2 & 2 & 3 & 3 & 3 & F 8 & 1 & 2 & 1 & 1 & 2 & 2 \\
\hline C 4 & 1 & 1 & 1 & 1 & 1 & 1 & F 9 & 2 & 2 & 1 & 2 & 2 & 2 \\
\hline C 5 & 1 & 1 & 1 & 1 & 1 & 1 & F 10 & 3 & 1 & 2 & 2 & 1 & 2 \\
\hline C 6 & 1 & 1 & 1 & 1 & 1 & 1 & & & & & & & \\
\hline C 7 & 1 & 1 & 1 & 1 & 1 & 1 & & & & & & & \\
\hline C 8 & 2 & 1 & 2 & 1 & 1 & 1 & & & & & & & \\
\hline C 9 & 2 & 2 & 1 & 1 & 2 & 1 & & & & & & & \\
\hline C 10 & 1 & 1 & 1 & 1 & 1 & 1 & & & & & & & \\
\hline C 11 & 1 & 1 & 1 & 1 & 1 & 1 & & & & & & & \\
\hline C 12 & 1 & 1 & 1 & 1 & 1 & 1 & & & & & & & \\
\hline C 13 & 1 & 1 & 1 & 1 & 1 & 1 & & & & & & & \\
\hline C 14 & 1 & 1 & 1 & 2 & 1 & 1 & & & & & & & \\
\hline D 1 & 1 & 1 & 1 & 1 & 1 & 1 & & & & & & & \\
\hline D 2 & 1 & 1 & 1 & 2 & 1 & 2 & & & & & & & \\
\hline D 3 & 1 & 2 & 1 & 2 & 2 & 2 & & & & & & & \\
\hline D 4 & 1 & 1 & 1 & 1 & 1 & 1 & & & & & & & \\
\hline D 5 & 1 & 1 & 1 & 1 & 1 & 1 & & & & & & & \\
\hline D 6 & 2 & 1 & 1 & 1 & 1 & 1 & & & & & & & \\
\hline D 7 & 2 & 1 & 2 & 1 & 1 & 1 & & & & & & & \\
\hline D 8 & 2 & 2 & 3 & 3 & 3 & 2 & & & & & & & \\
\hline D 9 & 1 & 1 & 1 & 1 & 1 & 1 & & & & & & & \\
\hline D 10 & 2 & 1 & 1 & 1 & 1 & 1 & & & & & & & \\
\hline D 11 & 1 & 1 & 1 & 1 & 1 & 1 & & & & & & & \\
\hline D 12 & 1 & 1 & 1 & 1 & 1 & 1 & & & & & & & \\
\hline D 13 & 1 & 1 & 1 & 1 & 1 & 1 & & & & & & & \\
\hline D 14 & 1 & 1 & 1 & 1 & 1 & 1 & & & & & & & \\
\hline
\end{tabular}


7-4. Algal cover

Table 7.

Coverage with macroalgae and the dominant macroalgal taxa in each quadrat (QD; Fig. 1d) during April or May of 2005 to 2010. Coverage (CV) was estimated visually at low tide, and assigned to one of the following four classes: $1(<25 \%), 2(25-50 \%), 3(50-75 \%)$, or $4(>75 \%)$. The dominant taxa (DT) are shown using the following abbreviations: $\mathrm{R}$ (red algae, Rhodophyta), B (brown algae, Phaeophyta), and G (green algae, Chlorophyta). The order of coverage by these taxa was determined intuitivelly in the field, and is shown using $>$. When two or three groups were equally abundant, the alphabetical abbreviations were punctuated. -, no data. 


\begin{tabular}{|c|c|c|c|c|c|c|c|c|c|c|c|c|}
\hline \multicolumn{13}{|c|}{ Table $7-1$} \\
\hline & 2005 & & 2006 & & 2007 & & 2008 & & 2009 & & 2010 & \\
\hline QD & $\mathrm{CV}$ & DT & $\mathrm{CV}$ & DT & $\mathrm{CV}$ & DT & CV & DT & $\mathrm{CV}$ & DT & CV & DT \\
\hline A 1 & 1 & G & 2 & G & 1 & G & 1 & $\mathrm{G}$ & 2 & G & 1 & $\mathrm{C}$ \\
\hline A 2 & 3 & G & 3 & G & 2 & $G>R$ & 4 & $\mathrm{G}>\mathrm{R}$ & 4 & $G>R$ & 2 & $\mathrm{G}>\mathrm{R}$ \\
\hline A 3 & 1 & G & 2 & G & 1 & $\mathrm{G}$ & 2 & $\mathrm{G}>\mathrm{R}$ & 2 & $G>R$ & 3 & $G>R$ \\
\hline A 4 & 1 & $\mathrm{R}>\mathrm{G}$ & 1 & $\mathrm{G}$ & 1 & $\mathrm{~B}>\mathrm{G}$ & 1 & $\mathrm{G}>\mathrm{R}$ & 2 & $G>B$ & 1 & $\mathrm{G}>\mathrm{R}$ \\
\hline A 5 & 1 & $\mathrm{G}$ & 2 & $\mathrm{G}$ & 2 & $C>B$ & 1 & $\mathrm{G}>\mathrm{R}$ & 7 & $G>R$ & 3 & $\mathrm{G}>\mathrm{R}$ \\
\hline A 6 & 1 & $\mathrm{G}, \mathrm{R}$ & 2 & $\mathrm{G}$ & 2 & $G>R$ & 1 & $\mathrm{G}>\mathrm{R}$ & & $G>R$ & 2 & $\mathrm{R}>\mathrm{B}$ \\
\hline A 7 & 1 & $\mathrm{R}$ & 1 & $\mathrm{R}$ & 1 & $\mathrm{R}>\mathrm{G}$ & 1 & $\mathrm{R}>\mathrm{G}$ & 1 & $\mathrm{R}>\mathrm{G}$ & 1 & $R>B$ \\
\hline A 8 & 1 & $\mathrm{~B}>\mathrm{R}$ & - & $\mathrm{R}>\mathrm{B}$ & 2 & $B>R$ & 2 & $\mathrm{G}>\mathrm{R}$ & 2 & $B>R$ & 2 & $\mathrm{~B}>\mathrm{R}$ \\
\hline B 1 & 1 & $\mathrm{G}$ & 1 & $\mathrm{G}$ & 1 & B & 2 & $\mathrm{G}$ & 0 & G & 3 & G \\
\hline B 2 & 2 & $\mathrm{G}$ & 1 & $\mathrm{G}$ & 2 & B & 2 & $\mathrm{G}>\mathrm{R}$ & 2 & $\mathrm{G}>\mathrm{R}$ & 2 & $\mathrm{G}>\mathrm{R}$ \\
\hline B 3 & 1 & $\mathrm{G}$ & 3 & G & 2 & $G>R$ & 2 & $\mathrm{G}>\mathrm{R}$ & 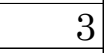 & $G>R$ & 1 & $G>R$ \\
\hline B 4 & 1 & $\mathrm{G}$ & 2 & $\mathrm{G}$ & 1 & $G>R$ & 1 & $\mathrm{G}>\mathrm{R}$ & 1 & $G>R$ & 2 & $G>R$ \\
\hline B 5 & 1 & 0 & 1 & G & 1 & $\mathrm{R}$ & 1 & $\mathrm{R}$ & 1 & $G>B$ & 1 & $G>R$ \\
\hline B 6 & 1 & $\mathrm{R}$ & 1 & G & 1 & $R>G$ & 1 & $G>R$ & 1 & $G>B$ & 1 & $G>R$ \\
\hline B 7 & 1 & G & 1 & $\mathrm{G}$ & 1 & G & 1 & $\mathrm{G}$ & 1 & $\mathrm{G}$ & 1 & $G>R$ \\
\hline B 8 & 1 & G & 1 & $\mathrm{G}$ & 1 & $\mathrm{G}$ & 1 & $\mathrm{G}$ & 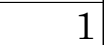 & $G>R$ & 1 & $\mathrm{G}>\mathrm{R}$ \\
\hline B 9 & 1 & $\mathrm{R}, \mathrm{B}$ & - & $\mathrm{R}$ & 1 & B & 1 & $\mathrm{R}>\mathrm{B}$ & 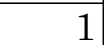 & $\mathrm{R}>\mathrm{G}$ & 1 & $\mathrm{R}>\mathrm{G}$ \\
\hline B 10 & 2 & $R>B$ & 2 & $\mathrm{R}$ & 1 & $\mathrm{R}>\mathrm{B}$ & 0 & $\mathrm{R}>\mathrm{B}$ & ? & $B>R$ & 2 & $R>B$ \\
\hline C 1 & 1 & $\mathrm{G}$ & - & $\mathrm{G}$ & 2 & $\mathrm{G}$ & 1 & $\mathrm{G}$ & . & $\mathrm{G}$ & 2 & $\mathrm{G}$ \\
\hline C 2 & 1 & $\mathrm{G}$ & 3 & $\mathrm{G}$ & 2 & $G>R$ & 2 & $\mathrm{G}>\mathrm{R}$ & 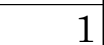 & $G>R$ & 1 & $G>R$ \\
\hline 3 & 1 & $\mathrm{G}$ & 2 & $\mathrm{G}$ & 1 & $G>R$ & 3 & $\mathrm{G}>\mathrm{R}$ & 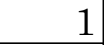 & $G>R$ & 1 & $G>R$ \\
\hline 4 & 1 & 0 & 1 & $\mathrm{G}$ & 1 & 0 & 7 & 0 & 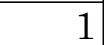 & $\mathrm{G}$ & 1 & $G>R$ \\
\hline 5 & 1 & $\mathrm{G}$ & 1 & $\mathrm{G}$ & 1 & $\mathrm{G}$ & 1 & $\mathrm{G}$ & 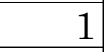 & $\mathrm{G}$ & 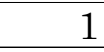 & $\mathrm{G}$ \\
\hline 6 & 1 & $\mathrm{G}$ & 1 & $\mathrm{G}$ & 1 & $G>R$ & 1 & $\mathrm{G}$ & 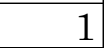 & $\mathrm{G}$ & 1 & $\mathrm{G}$ \\
\hline 7 & 1 & G & 1 & $\mathrm{G}$ & 1 & $G>R$ & 1 & $\mathrm{G}$ & 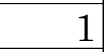 & $\mathrm{G}$ & 1 & G \\
\hline 8 & 1 & G & 1 & G & 1 & $G>R$ & 1 & $\mathrm{G}>\mathrm{R}$ & 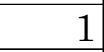 & $G>R$ & 1 & $G>R$ \\
\hline 9 & 1 & $R>G$ & - & $\mathrm{R}$ & 1 & G & 1 & $\mathrm{G}>\mathrm{R}$ & 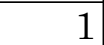 & $G>R$ & 1 & $R>G$ \\
\hline 10 & 1 & $\mathrm{R}$ & 1 & $\mathrm{R}$ & 1 & $\mathrm{R}>\mathrm{G}$ & 1 & $\mathrm{G}>\mathrm{R}$ & . & $\mathrm{R}>\mathrm{G}$ & 1 & $\mathrm{R}>\mathrm{G}$ \\
\hline 11 & 3 & $\mathrm{~B}>\mathrm{R}$ & 3 & $\mathrm{R}, \mathrm{B}$ & 3 & $R>B$ & 1 & $\mathrm{~B}>\mathrm{R}$ & . & $B>R$ & 2 & $R>B$ \\
\hline C 12 & 1 & B & 1 & B & 1 & $\mathrm{G}, \mathrm{B}, \mathrm{R}$ & 1 & $\mathrm{~B}>\mathrm{R}$ & . & $\mathrm{R}>\mathrm{G}$ & 1 & $\mathrm{R}>\mathrm{G}$ \\
\hline C 13 & 2 & B & 1 & B & 2 & $B>R$ & 1 & $\mathrm{~B}>\mathrm{R}$ & 1 & $B>R$ & 2 & $\mathrm{~B}>\mathrm{R}$ \\
\hline C 14 & 1 & $\mathrm{~B}>\mathrm{R}$ & 2 & $\mathrm{R}$ & 2 & $R>B$ & 4 & $\mathrm{~B}>\mathrm{R}$ & 2 & $\mathrm{~B}>\mathrm{R}$ & 2 & $R>B$ \\
\hline D 1 & 1 & G & 1 & G & 1 & G & 4 & $\mathrm{G}$ & 7 & $\mathrm{G}$ & 2 & $\mathrm{G}$ \\
\hline D 2 & 1 & $\mathrm{G}$ & 1 & $\mathrm{G}$ & 1 & $\mathrm{G}$ & 7 & $\mathrm{G}$ & 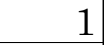 & $\mathrm{G}$ & 1 & $\mathrm{G}$ \\
\hline D 3 & 1 & $\mathrm{G}$ & 1 & $\mathrm{G}$ & 1 & $\mathrm{G}$ & 1 & $\mathrm{G}$ & - & $\mathrm{G}$ & 3 & $\mathrm{G}$ \\
\hline D 4 & 1 & $\mathrm{G}$ & 1 & $\mathrm{G}$ & 1 & $\mathrm{G}$ & 1 & $\mathrm{G}$ & 1 & $\mathrm{G}$ & 2 & $\mathrm{G}$ \\
\hline D 5 & 1 & $\mathrm{G}$ & 1 & G & 1 & $G>R$ & 1 & $\mathrm{G}>\mathrm{R}$ & 2 & $G>R$ & 1 & $\mathrm{G}$ \\
\hline D 6 & 1 & $\mathrm{G}$ & 1 & $\mathrm{G}$ & 1 & $\mathrm{G}, \mathrm{R}$ & 1 & $\mathrm{G}$ & 2 & $\mathrm{G}$ & 1 & G \\
\hline D 7 & 2 & $\mathrm{G}$ & 1 & $\mathrm{G}$ & 3 & $G>R$ & 2 & $\mathrm{G}$ & 3 & $G>R$ & 1 & $G>R$ \\
\hline D 8 & 1 & G & 1 & $R>G$ & 2 & $G>R$ & 2 & $G>R$ & 3 & $G>R$ & 3 & $G>R$ \\
\hline D 9 & 1 & $\mathrm{R}, \mathrm{B}, \mathrm{G}$ & 1 & $\mathrm{R}, \mathrm{B}$ & 1 & $G>R$ & 2 & $G>B$ & 2 & $G>R$ & 2 & $G>R$ \\
\hline D 10 & 1 & $\mathrm{R}$ & 1 & $\mathrm{R}$ & 1 & $\mathrm{R}$ & 1 & $\mathrm{R}>\mathrm{G}$ & 2 & $G>R$ & 1 & $\mathrm{R}$ \\
\hline D 11 & 1 & B & 2 & $\mathrm{R}, \mathrm{B}$ & 1 & $\mathrm{R}$ & 1 & $\mathrm{~B}>\mathrm{R}$ & 4 & $B>R$ & 1 & $R>B$ \\
\hline D 12 & 1 & $R, B$ & 1 & $\mathrm{R}, \mathrm{B}$ & 1 & $B>R$ & 1 & $\mathrm{~B}>\mathrm{R}$ & 1 & $B>R$ & 1 & $\mathrm{~B}>\mathrm{R}$ \\
\hline D 13 & 1 & B & 1 & $B>R$ & 1 & $R>B$ & 1 & B & 1 & $B>R$ & 1 & $B>R$ \\
\hline D 14 & 3 & $\mathrm{R}, \mathrm{B}$ & 3 & $R>B$ & 3 & $\mathrm{R}>\mathrm{B}$ & 4 & $\mathrm{R}>\mathrm{B}$ & 4 & $R>B$ & 4 & $R>B$ \\
\hline
\end{tabular}




\begin{tabular}{|c|c|c|c|c|c|c|c|c|c|c|c|c|}
\hline \multicolumn{13}{|c|}{ Table 7-2 } \\
\hline & 2005 & & 2006 & & 2007 & & 2008 & & 2009 & & 2010 & \\
\hline $\mathrm{QD}$ & $\mathrm{CV}$ & DT & $\mathrm{CV}$ & DT & $\mathrm{CV}$ & DT & $\mathrm{CV}$ & DT & $\mathrm{CV}$ & DT & $\mathrm{CV}$ & DT \\
\hline E 1 & 1 & 0 & 1 & $\mathrm{G}$ & 1 & $\mathrm{G}$ & 1 & 0 & 1 & 0 & 1 & $\mathrm{G}$ \\
\hline E 2 & 1 & $\mathrm{G}$ & - & G & 1 & $\mathrm{G}$ & 1 & 0 & 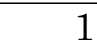 & 0 & 1 & $\mathrm{G}$ \\
\hline E 3 & 1 & $\mathrm{G}$ & - & $\mathrm{G}$ & 1 & $\mathrm{G}$ & 1 & 0 & 1 & $\mathrm{G}$ & 1 & $\mathrm{G}$ \\
\hline E 4 & 1 & $\mathrm{G}$ & 1 & $\mathrm{G}$ & 1 & $\mathrm{G}>\mathrm{R}$ & 1 & $\mathrm{G}$ & 1 & $\mathrm{G}$ & 2 & $\mathrm{G}$ \\
\hline E 5 & 1 & $\mathrm{G}$ & 1 & $\mathrm{R}>\mathrm{G}$ & 2 & $\mathrm{G}>\mathrm{R}$ & 2 & $\mathrm{G}, \mathrm{R}$ & 3 & $G>R$ & 3 & $\mathrm{G}$ \\
\hline E 6 & 1 & $\mathrm{G}$ & 2 & $R, B$ & 2 & $\mathrm{G}>\mathrm{R}$ & 2 & $\mathrm{G}>\mathrm{R}$ & 3 & $G>R$ & 3 & $G>R$ \\
\hline E 7 & 1 & $\mathrm{G}$ & 1 & $\mathrm{G}$ & 3 & $\mathrm{G}>\mathrm{R}$ & 2 & $G>B$ & - & $G>R$ & 1 & $G>R$ \\
\hline E 8 & 3 & $\mathrm{R}>\mathrm{G}$ & 3 & $\mathrm{R}, \mathrm{B}$ & 3 & $\mathrm{R}>\mathrm{G}$ & 1 & $\mathrm{G}>\mathrm{B}$ & 2 & $G>R$ & 3 & $\mathrm{R}>\mathrm{G}$ \\
\hline E 9 & 1 & $\mathrm{R}>\mathrm{B}$ & 2 & $R>B$ & 2 & $\mathrm{R}>\mathrm{B}$ & 2 & $\mathrm{~B}>\mathrm{G}$ & 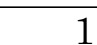 & $\mathrm{R}>\mathrm{G}$ & 2 & $\mathrm{R}>\mathrm{G}$ \\
\hline E 10 & 1 & $\mathrm{R}$ & 2 & $\mathrm{R}>\mathrm{B}$ & 3 & $\mathrm{R}>\mathrm{G}$ & 2 & $\mathrm{R}>\mathrm{B}$ & 1 & $\mathrm{R}>\mathrm{B}$ & 2 & $\mathrm{R}>\mathrm{G}$ \\
\hline E 11 & 3 & $\mathrm{~B}>\mathrm{R}$ & - & $\mathrm{B}$ & 2 & $\mathrm{~B}>\mathrm{R}$ & 2 & $\mathrm{~B}>\mathrm{R}$ & 3 & $\mathrm{~B}>\mathrm{R}$ & 2 & $\mathrm{~B}>\mathrm{R}$ \\
\hline E 12 & 3 & $\mathrm{~B}$ & 4 & $\mathrm{~B}$ & 2 & $\mathrm{~B}>\mathrm{R}$ & 1 & $\mathrm{~B}>\mathrm{R}$ & 3 & $\mathrm{~B}>\mathrm{R}$ & 3 & $\mathrm{~B}>\mathrm{R}$ \\
\hline E 13 & 3 & B & 2 & B & 2 & $\mathrm{~B}>\mathrm{R}$ & 2 & $\mathrm{~B}>\mathrm{R}$ & - & - & 2 & $\mathrm{~B}>\mathrm{R}$ \\
\hline F 1 & 1 & $\mathrm{G}$ & 1 & $\mathrm{G}$ & 1 & $\mathrm{G}$ & 1 & $\mathrm{G}$ & 1 & $\mathrm{G}$ & 2 & $\mathrm{G}>\mathrm{R}$ \\
\hline $\mathrm{F} 2$ & 1 & 0 & 1 & $\mathrm{G}>\mathrm{C}$ & 1 & $\mathrm{G}$ & 1 & $\mathrm{G}$ & 1 & $\mathrm{G}$ & 2 & $\mathrm{G}>\mathrm{R}$ \\
\hline F 3 & 1 & $\mathrm{R}$ & 1 & $C>R$ & 1 & $\mathrm{R}$ & 1 & $\mathrm{G}>\mathrm{R}$ & 1 & $\mathrm{G}, \mathrm{R}$ & 2 & $\mathrm{G}>\mathrm{R}$ \\
\hline F 4 & 2 & $\mathrm{R}$ & 2 & $\mathrm{R}>\mathrm{C}$ & 2 & $\mathrm{R}>\mathrm{G}$ & 2 & $R>B$ & 2 & $\mathrm{G}, \mathrm{R}$ & 2 & $G>R$ \\
\hline F 5 & 1 & $\mathrm{R}$ & 1 & $\mathrm{R}>\mathrm{G}$ & 2 & $\mathrm{R}>\mathrm{G}$ & 2 & $\mathrm{G}>\mathrm{R}$ & 2 & $G>R$ & 1 & $G>R$ \\
\hline F 6 & 1 & $\mathrm{R}$ & 1 & $G>R$ & 1 & $\mathrm{G}>\mathrm{R}$ & 1 & $\mathrm{G}>\mathrm{R}$ & 2 & $\mathrm{G}, \mathrm{R}$ & 2 & $R>G$ \\
\hline F 7 & 1 & $\mathrm{R}$ & 1 & $\mathrm{G}>\mathrm{R}$ & 1 & $\mathrm{G}>\mathrm{R}$ & 1 & $\mathrm{G}>\mathrm{R}$ & 2 & $G>R$ & 1 & $\mathrm{~B}>\mathrm{R}$ \\
\hline F 8 & 2 & $\mathrm{~B}>\mathrm{R}$ & 3 & $B>R$ & 2 & $\mathrm{~B}>\mathrm{R}$ & 2 & $\mathrm{~B}>\mathrm{R}$ & 2 & $B>R$ & 3 & $R>B$ \\
\hline F 9 & 2 & B & 3 & $B>R$ & 2 & $B>R$ & 2 & $\mathrm{~B}>\mathrm{R}$ & 2 & $B>R$ & 3 & $B>R$ \\
\hline F 10 & 4 & $\mathrm{~B}>\mathrm{R}$ & 3 & $\mathrm{~B}>\mathrm{G}$ & 2 & $\mathrm{~B}>\mathrm{R}$ & 3 & $\mathrm{~B}>\mathrm{R}$ & 2 & $B>R$ & 3 & $\mathrm{~B}>\mathrm{R}$ \\
\hline
\end{tabular}


7-5. Climatic and oceanographic data

Table 8.

Summary of the oceanographic data from 1984 to 2010 around the study site. AT, the monthly mean air temperature based on the values measured daily at St. 1 (Fig. 1b). Typhoon, the number of typhoons that entered within $1000 \mathrm{~km}$ and $200 \mathrm{~km}$ (in brackets) circles of the study site during April to November each year. These data were obtained from the website of the Japan Meteorological Agency (http://www.data.jma.go.jp). Nearshore WT, monthly mean of the water temperature measured daily at St. 2 (Fig. 1b) by the Seto Marine Biological Laboratory of Kyoto University. Offshore WT, water temperature measured once a month at $50 \mathrm{~m}$ depth at 9 offshore stations $(2-20 \mathrm{~km}$ from the coast, cross marks in Fig. 1a). Sal, salinity at $10 \mathrm{~m}$ depth at St. 3 (Fig. 1b). The offshore temperature and salinity were measured once a month by the Fisheries Experimental Station of Wakayama Prefecture. Kuroshio, the annual mean distance of the Kuroshio Current from Cape Shionomisaki (Fig. 1a) reported by the Maritime Safety Agency of Japan. COD, SS, T-N (total nitrogen), and T-P (total phosphorous) were measured at the surface layer of six points around St 4 (Fig. 1b) once a year between July and September by the Shirahama Municipality Government, and the mean of the six values is shown for each parameter. -, no data. 


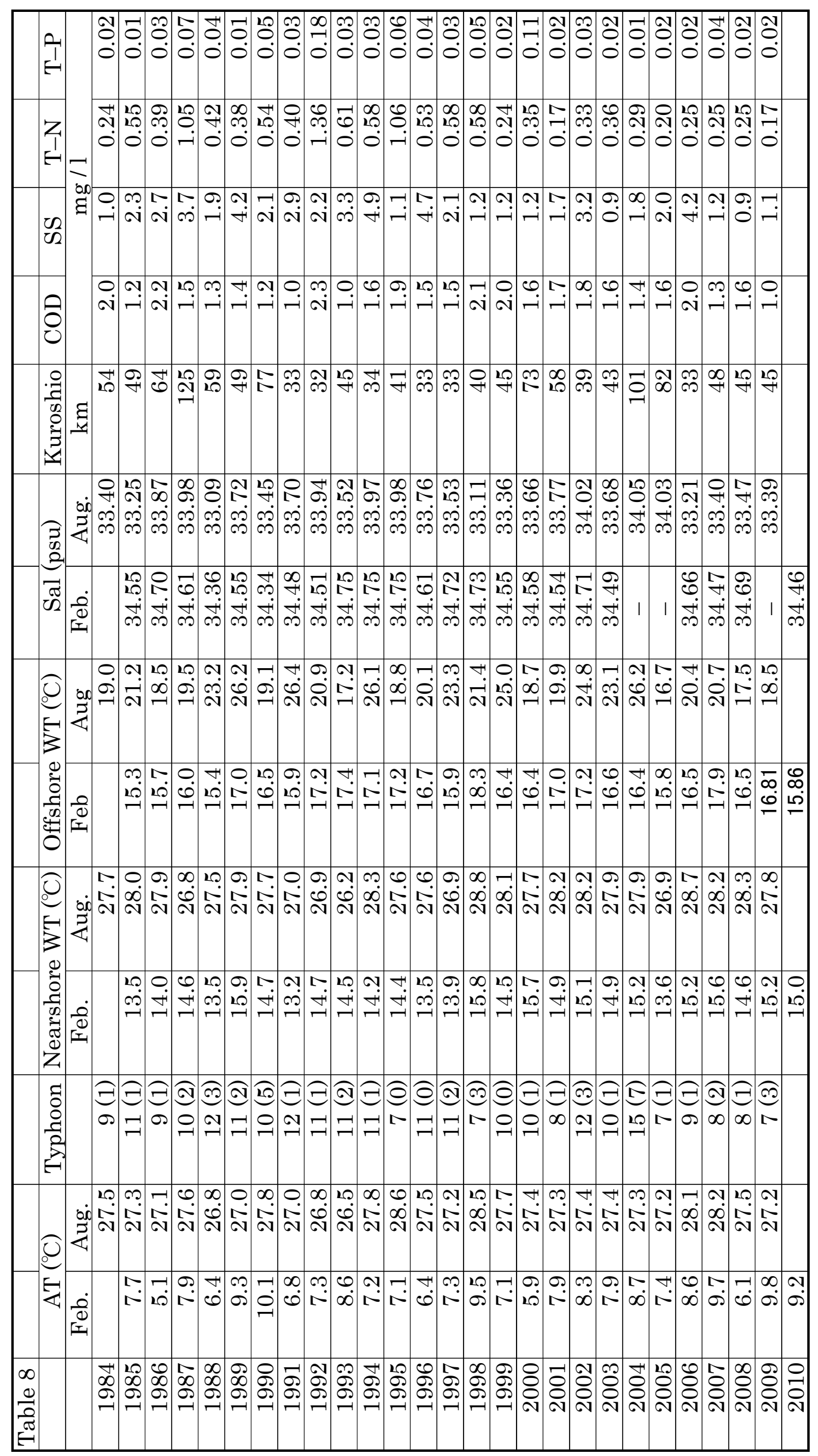

